\title{
ON A RIEMANNIAN METHOD IN QUANTUM THEORY ABOUT CURVED SPACE-TIME*)
}

\author{
A. Uhlmann \\ Department of Physics and NTZ, Karl Marx University, Leipzig, GDR
}

\begin{abstract}
We start from a given Lorentz metric and a vector field of world lines along which observers and measure devices may move. We describe a procedure to associate one-particle Hilbert spaces and one-particle Hamiltonians to space-like hypersurfaces using a transition to a Riemannian metric. With the aid of suitable boundary conditions one can confine the particle within a world tube ("box quantization about curved space-time manifolds").
\end{abstract}

Our aim is to treat a rather restricted problem in quantizing about curved spacetime manifolds concerning Klein-Gordon particles, in particular their one-particle states. In this approach $[1,2]$, see also [3], we try to follow the spirit of Euclidean quantum field theory as described, for example, in [4].

However, the lack of any symmetry in the general case forces us to reformulate this approach in terms of potential theoretic concepts without using either symmetries or analytic continuations. Aiming even at a sort of box quantization we cannot rely on the Cauchy completeness of the hypersurfaces involved either. These features distinguish the following from other treatments rather clearly. (For an overview of other methods, most of them much more ambitious, see $[3,5,6]$.)

The presentation in this paper deviates, influenced by [7], at one point from [1,2], though this deviation does not affect the stationary case, which as is well known, may be treated by a couple of methods. The merit of this change is that now all the physical quantities depend, explicitly, and implicitly only on the systems and the observers past - a feature which is even in the Euclidean theory a rather implicit truth.

Let $M$ be a 4-manifold and $g_{i k}$ a Lorentz metric on $M$, the diagonal form of which may have signs $+\longrightarrow$.

On $M$ we consider a time-like, forward directed, and normalized vector field

$$
\left\{e^{j}\right\}, \quad g_{i k} e^{i} e^{j}=1 .
$$

This vector field may be interpreted as the 4-velocities belonging to some set of observers, measure devices, moving along world lines given by the solutions of

$$
t \rightarrow y(t), \quad \mathrm{d} y^{i} / \mathrm{d} t=e^{i} .
$$

A box quantization is defined by a world tube $T$ outside of which there is a potential

*) Dedicated to Professor Ivan Úlehla on the occasion of his sixtieth birthday. 
energy of infinite height preventing the particle and the observers to escape out of the tube $T$. We assume the tube to be an open, connected subset of $M$ with the property: An integral curve of (2) is either contained in the tube completely, or the intersection of it with the tube is empty. We need further a fibring of $T$ by relatively open pieces $V_{s}$ of space-like hypersurfaces, $s$ a real parameter.

We demand:

(3) (i) $V_{s} \cap V_{s^{\prime}}=$ empty if $s \neq s^{\prime}$.

(ii) $V_{s}$ depends causally on $V_{t}$ if $s>t$.

(iii) Every integral curve of (2), contained in $T$, intersects every $V_{s}$.

(iv) $T$ is the union of all the $V_{\mathrm{s}}$,

It is useful to define

$$
T_{s}=\left\{\text { union. of all } V_{t} \text { with } t \leqq s\right\}
$$

in particular,

$$
T=T_{\infty} .
$$

Next we have to consider an auxiliary metric $\tilde{g}_{i k}$ given by

$$
g_{i k}+\tilde{g}_{i k}=2 e_{i} e_{k} .
$$

The metric $\tilde{g}_{i k}$ is a Riemannian one. As a matter of fact

$$
e_{i}=g_{i k} e^{k}=\tilde{g}_{i k} e^{k}
$$

Further, the four-volumes $\mathrm{d}^{4} v$ of the two metrics appearing in (6) coincide.

We shall consider the Laplace-Beltrami operator

$$
\tilde{\Delta}
$$

with respect to $\tilde{g}_{i k}$.

Let $u$ be a regular function with compact support and

$$
\operatorname{supp} u \subseteq T_{s} \text {. }
$$

We shall consider solutions $f$ of

$$
\left(-\tilde{\Delta}+m^{2}\right) f=u
$$

where $m$ is the rest mass of Klein-Gordon particles. We need two different solutions of (10) which we describe now. At first let $f$ satisfy the following Dirichlet condition: For every $\varepsilon>0$ the set $|f| \geqq \varepsilon$ is a compact set consisting of interior points of $T_{\text {s }}$ only. This and (10) uniquely define $f$ and we write

$$
f=G_{s}^{D} u .
$$


Next we define $G_{s}^{N}$ by requiring for $f$

$$
\tilde{n}^{j} \frac{\partial}{\partial x^{j}} f=0 \text { on } V_{s}
$$

where $\tilde{n}^{j}$ is the normal vector of $V_{s}$ with respect to $\tilde{g}_{i k}$, and furthermore, that the set $|f| \geqq \varepsilon$ for every $\varepsilon>0$ is contained in the set $V_{s} \cup$ (interior points of $T_{s}$ ). Then

$$
f=G_{s}^{N} u
$$

denotes the solution of $(10)$ respecting the just mentioned boundary conditions.

We are now prepared to consider the Dirichlet integrals

$$
D(f, g)_{s}=\int_{T_{s}}\left\{\bar{f}_{, i} g^{i k} g_{, \psi}+m^{2} \bar{f} g\right\} \mathrm{d}^{4} v,
$$

We need some general inequalities. If

$$
g_{s}=G_{s}^{N} u, \quad f_{s}=G_{s}^{D} u, \quad \operatorname{supp} u \subseteq T_{s},
$$

one knows

$$
D\left(f_{t}, f_{t}\right)_{t} \leqq D\left(g_{s}, g_{s}\right)_{s} \text { if } t \geqq s
$$

and again fot $t \geqq s$,

$$
D\left(f_{t}, f_{t}\right)_{t} \geqq D\left(f_{t}, f_{t}\right)_{s} \geqq D\left(f_{s}, f_{s}\right)_{s} .
$$

(17) is the well-known monotonicity of the Dirichlet problem by enlarging the domain for $g_{s}$ minimizes the Dirichlet integral. (16) is derivable from

$$
D\left(f_{t}, f_{s}\right)=\int_{t} \bar{f}_{t} u \mathrm{~d}^{4} v=D\left(f_{t}, f_{t}\right)_{t} .
$$

Indeed, using $D\left(f_{t}-g_{s}, f_{t}-g_{s}\right)_{s} \geqq 0$ one gets from $(*)$

$$
D\left(g_{s}, g_{s}\right)_{s} \geqq 2 D\left(f_{t}, f_{t}\right)_{t}-D\left(f_{t}, f_{t}\right)_{s} \geqq D\left(f_{t}, f_{t}\right)_{t}
$$

where (17) has been used in the last step.

Our next task is to define a Hilbert space $N_{s}$ as follows: $N_{s}$ is the completion in norm of all regular functions $u$ with supp $u \subseteq T_{s}$ by the scalar product

$$
\left(u_{1}, u_{2}\right)_{s}=\frac{1}{2} D\left(G_{s}^{N} u_{1}, G_{s}^{N} u_{2}\right)_{s} .
$$

According to the inequality (16) the sesquilinear form

$$
\left\langle u_{1}, u_{2}\right\rangle_{t}=D\left(G_{t}^{D} u_{1}, G_{t}^{D} u_{2}\right)_{t}
$$

is bounded and positive semidefinite on a dense domain of the Hilbert space $N_{s}$ for $s \leqq t$. Hence there exists a bounded and positive semidefinite operator $E_{s, t}$ 
acting on $N_{s}$ and satisfying

$$
\left\langle u_{1}, u_{2}\right\rangle_{t}=\left(u_{1}, E_{s, t} u_{2}\right)_{s} .
$$

Furthermore, as a consequence of (17), we get

$$
E_{s, t} \geqq E_{s, r} \text { if } \quad s \leqq r \leqq t .
$$

All this is the prelude. We are now going to construct one-particle Hilbert spaces sitting on the hypersurface pieces $V_{s}$. If $r<s$, it is obvious from potential theory that there are elements of $N_{s}$ representable by distributions the support of which is contained within $V_{r}$. There is a closed subspace, $N_{s, r}$, of $N_{s}$ consisting of all distributions

$$
h \rightarrow u(h)=\int h \hat{u} \mathrm{~d}^{3} v
$$

where the integral is extended over $V_{r}$, and $\hat{u}$ is a measurable function on $V_{r}$ satisfying certain growth conditions. $\mathrm{d}^{3} v$ is the three-volume induced by the metric on $V_{r}$. However, because we have assumed Neumann conditions on $V_{s}$, the same is true for the limit $N_{s, r} \rightarrow N_{s, s}$, where the distributions concentrated on $V_{r}$ have been transported along the world lines (2) of the observers. We define

$$
F_{s}=N_{s, s}
$$

and we denote by $P_{s}$ the orthoprojection of $N_{s}$ onto $F_{s} . F_{s}$ is the one-particle Hilbert space for a neutral scalar particle at the "instant" $V_{s}$ in the Tomonaga-Schwinger sense by definition.

From (21) it is seen that

$$
P_{s} E_{s, t} P_{s} \geqq P_{s} E_{s, r} P_{s} \text { if } s \leqq r \leqq t
$$

Now in a stationary metric and with $e^{i}$ parallel to the Killing field intersecting the submanifolds $V_{s}$ orthonormally, the operator (24) may be viewed as

$$
P_{s} E_{s, t} P_{s}=1-\exp \left(-(t-s) H_{s}\right)
$$

with $H_{s}$ being the one-particle hamiltonian. Furthermore, one can identify all the $F_{s}$ and all the $H_{s}$ with different $s$. Generally, however, (24) cannot be connected with a semigroup due to non-stationarity of the metric. Therefore we have to define

$$
H_{s}=\lim _{t \rightarrow+s}(t-s)^{-1}\left(P_{s} E_{s, t} P_{s}-P_{s} E_{s, s} P_{s}\right)
$$

accordingly. $F_{s}$ is concentrated on $V_{s}$, and $E_{s, s}$ demands for Dirichlet boundary conditions. From this one infers

$$
P_{s} E_{s, s} P_{s}=0 \text {. }
$$


Thus

$$
H_{s}=\lim _{t \rightarrow+s}(t-s)^{-1} P_{s} E_{s, t} P_{s}
$$

where the domain of strong convergence of (27) is the domain of definition of $H_{s}$. Clearly, and in the Euclidean-Riemannian treatments trivially, the spectrality requirement

$$
H_{s} \geqq 0
$$

is satisfied guaranteeing stability at every "instant of time" $V_{s}$, but of course this does not imply stability in the flow of time, giving possibilities for particle creation processes.

Remark: The constructions above may not work without modification if the boundary of $T$ is touching an horizon of a singularity. For then $g_{i k}$ may become degenerate or not regular enough to pass all the needed arguments, in particular this concerns the manipulation $(*)$ and the solutions of the boundary problems. From $(*)$ there may arise extra terms. Concerning the solutions of equation (10) there seems to be no general theory in case the boundary is not regular enough.

Having constructed $F_{s}$ and $H_{s}$ let us see how it works for stationary metrics. Let us use a coordinate system $\left\{x^{0}=t, x^{1}, x^{2}, x^{3}\right\}$ a metric $\mathrm{d} r^{2}$ depending only on $x^{1}$, $x^{2}$, and $x^{3}$ such that

$$
\mathrm{d} s^{2}=g_{00} \mathrm{~d} t^{2}-\mathrm{d} r^{2}
$$

where $g_{00}$ does not depend on $t$. $V_{s}$ shall be given by the equation $t=s$, locally, and in this frame.

$$
e_{0}=\left(g_{00}\right)^{1 / 2}, \quad e_{1}=e_{2}=e_{3}=0
$$

We need the reflection operator $R_{s}$ relative to $V_{s}$

$$
R_{s} f\left(t, x^{1}, x^{2}, x^{3}\right)=f\left(s-t, x^{1}, x^{2}, x^{3}\right)
$$

which enables us to express all the occurring Dirichlet integrals in terms of (19) for $t=\infty$, i.e.

$$
\left\langle u_{1}, u_{2}\right\rangle_{\infty} \text {. }
$$

The reflection $R_{s}$ is an isometry of the original and of that metric introduced by (6). It commutes with the Laplace-Beltrami operator.

Suppose now supp $u \subseteq T_{s}$. Then $G_{\infty}^{D}\left(u+R_{s} u\right)$ will have vanishing normal derivatives on $V_{s}$. Thus, on $V_{s}$, this expression coincides with $G_{s}^{N} u$. Therefore

$$
2\left(u_{1}, u_{2}\right)_{s}=\frac{1}{2}\left\langle u_{1}+R_{s} u_{2}, u_{1}+R_{s} u_{2}\right\rangle_{\infty}
$$

provided the carriers of $u_{1}, u_{2}$ are contained within $T_{s}$. This is due to (14) and the fact that $V_{s}$ divides $T$ into two parts which are isometric images one of another by $R_{s^{*}}$ In 


\section{A. Uhlman: On a Riemannian method...}

going to $F_{s}$ we obtain $R_{s} u=u$, and hence

$$
\left(u_{1}, u_{2}\right)_{s}=\left\langle u_{1}, u_{2}\right\rangle_{\infty} \text { for } u_{j} \in F_{s} .
$$

In the papers $[1,2],(32)$ has been used as a definition. This seems to be unphysical for non-stationary metrics. In the modified approach presented here, the s-independence of the scalar product is completely due to the stationarity of the metric.

We now use the reflection operator again supposing supp $u \subseteq T_{s}$. The expression $G_{\infty}^{D}\left(u-R_{s} u\right)$ fulfils Dirichlet conditions on $T_{s}$. It coincides on $T_{s}$ with $G_{s}^{D} u$, therefore. We get

$$
\left\langle u_{1}, u_{2}\right\rangle_{s}=\frac{1}{2}\left\langle u_{1}-R_{s} u_{2}, u_{1}-R_{s} u_{2}\right\rangle_{\infty}=\left\langle u_{1}, u_{2}\right\rangle_{\infty}+\left\langle u_{1}, R_{s} u_{2}\right\rangle_{\infty}
$$

an equation which, if combined with (17), expresses what is called "reflection positivity".

Assuming now $u_{j} \in F_{s}$ and $s \leqq t$ we get from the definitions

$$
\begin{aligned}
2\left(u_{1}, H_{s} u_{2}\right)_{s} & =\lim (t-s)^{-1}\left\{\left\langle u_{1}, u_{2}\right\rangle_{\infty}-\left\langle u_{1}, R_{t} u_{2}\right\rangle_{\infty}\right\}= \\
& =-(\mathrm{d} / \mathrm{d} t)\left(u_{1}, R_{t} u_{2}\right)_{s} \text { at } t=+s
\end{aligned}
$$

where a further simplification can be achieved by (32). The factor 2 in front of (34) is due to the fact that a reflection $R_{s}$ is equivalent to a transport along the Killing field by an amount of $2(t-s)$ provided $u \in F_{s}$ and $s \leqq t$.

The explicit calculation is now straightforward and we only describe the result. The Laplace-Beltrami operator can be written as

$$
\tilde{\Delta}=g^{00} \frac{\partial^{2}}{\partial t^{2}}+K
$$

where $K$ does not depend on $t$ and is elliptic on every $V_{s}$. Using the Hilbert space

$$
L^{2}\left(V_{s}, g^{1 / 2} \mathrm{~d} x^{1} \mathrm{~d} x^{2} \mathrm{~d} x^{3}\right)
$$

where $g$ is the determinant of the 4-metric (and not of $\left.\mathrm{d} r^{2} !\right)$ we define a self-adjoint extension $K^{D}$ of $K$ imposing Dirichlet boundary conditions on $V_{s}$. In (36) there is a unique self-adjoint and positive operator $H=H_{s}$ satisfying

$$
H^{2}=g_{00}^{1 / 4}\left(-K^{D}+m^{2}\right) g_{00}^{1 / 4} .
$$

Let us now consider a state vector

$$
u=w\left(x^{1}, x^{2}, x^{3}\right) \delta(t-s), \quad u \in F_{s} .
$$

Then we get

$$
(u, u)_{s}=\pi \int\left(\bar{w} H^{-1} w\right) g^{1 / 2} \mathrm{~d}^{3} x
$$


with $g$ being again the 4-determinant. One sees that the index $s$ does not play any important role in the stationary case. It is easy to see now the correctness of the identification

$$
H_{s}=H .
$$

Remark: As a matter of fact $(u, u)_{s}$ is given for the Minkowski metric $\mathrm{d} s^{2}$ by

$$
\int \bar{u}(\mathbf{x}) u(\mathbf{y}) W_{2}(\mathbf{y}-\mathbf{x}) \mathrm{d}^{4} x \mathrm{~d}^{4} y
$$

where $W_{2}$ denotes the two-point Wightman function, provided $u$ is of the form (38).

At the end we point a class of metrics for which the results of our procedure cannot be gained by any analytic continuation. They are given by

$$
\mathrm{d} t^{2}-S(t)^{2} \mathrm{~d} r^{2}
$$

where again $\mathrm{d} r^{2}$ is a 3-metric not depending on $t$. We choose $V_{s}$ orthogonal to $\left\{e^{i}\right\}=$ $=\{1,0,0,0\}$, i.e. as $t=$ const. This problem appears in the Klein-Gordon problem with Robertson-Walkers-type metrics.

Let us identify $\Delta_{3}$ with the Dirichlet self-adjoint extension of the Laplace-Beltrami operator belonging to the metric $\mathrm{d} r^{2}$ within the Hilbert space $F_{s}$.

Then we have the following fact in our procedure: $H_{s}$ commutes strongly with $\Delta_{3}$. For compact manifolds or essentially bounded ones ("box quantization") the eigenfunctions of $\Delta_{3}$ and $H_{s}$ coincide. The eigenvalues of $H_{s}$, however, depend in a complicated manner on the world time instant $t$. Their calculation depends on the behaviour of Green functions of a certain second order ordinary differential equation, allowing in principle numerical computations.

Received 18. 5. 1981.

\section{References}

[1] Uhlmann A.: On Quantization in Curved Space Time. In: Proc. of the Int. Workshop on High Energy Physics and Quantum Field Theory, Serpukhov, 1979.

[2] Uhlmann A.: Quantization method using Dirichlet's principle. Abstract Volume III of GR 9, Jena, 1980.

[3] Gibbons G. W.: Quantization about classical background metric. To appear in: Proceedings of GR 9, Jena.

[4] Simon B.: The $P_{2}$ Euclidean (Quantum) Field Theory. Princeton University Press, 1974.

[5] De Witt B. S.: Phys. Reports 19C (1975) 295.

[6] Isham C. J.: Quantum Gravity - An Overview. ICTP (79-80) 16.

[7] Glimm J., Jaffe A.: A Note on Reflection Positivity. HUT 79-B 71. 